\title{
Desenvolvimento da habilidade na escrita e a produção científica: cursos são necessários?
}

\author{
Writing skills development and scientific production: are needed \\ courses?
}

\section{El desarrollo de la habilidad en la escritura y la producción científica: ¿son necesarios cursos?}

\author{
Angela Maria Belloni Cuenca | abcuenca@usp.br \\ Universidade de São Paulo, Faculdade de Saúde Pública. São Paulo, Brasil. \\ Dulcenéa de Paula |dulcenea.paula@usp.br \\ Universidade de São Paulo, Faculdade de Saúde Pública. São Paulo, Brasil. \\ Ivan França Junior Iifjunior@usp.br \\ Universidade de São Paulo, Faculdade de Saúde Pública. São Paulo, Brasil.
}

\section{Resumo}

Este artigo é resultado de um estudo que tem como objetivo identificar se o aprimoramento do conhecimento sobre a produção de artigos científicos, reforçado por cursos de escrita científica, vem facilitando ao pesquisador (autor) a publicação de seus artigos. Entendemos que a relevância do estudo se deve ao reconhecimento da importância de investimentos no aprimoramento do pesquisador para a divulgação científica. Foi identificada a produção bibliográfica de egressos de cursos de aprimoramento da habilidade na escrita científica e comparada com o percurso de publicações desse egresso, antes e depois do curso. Foram utilizadas duas fontes de informação de acesso público para identificar a produção bibliográfica, científica e técnica, do pesquisador: currículo na Plataforma Lattes do CNPq; e fontes de dados bibliográficos: Lilacs e PubMed. Observou-se que o número de artigos publicados foi maior após a participação em um dos cursos e que, embora outros fatores influenciem na produção, iniciativas dessa natureza estimulam a divulgação científica.

Palavras-chave: comunicação e divulgação científica; alfabetização científica; cursos modulados; redação de artigos; saúde pública.

\begin{abstract}
This paper is product of a study to identify if the improvement in knowledge about writing scientific papers reinforced by writing courses had facilitated the publication by researcher (author) of his articles. We understand the relevance of the study is due to the recognition of the importance of investments in training courses for researchers to improve the scientific dissemination of their papers. We identified the bibliographic production of the participants in courses on scientific writing, and then we compared it with the number of publications by then prior to and after the course. We searched two public information
\end{abstract}


sources to identify the scientific and technical bibliographic production by researcher: curriculum in the Plataforma Lattes do CNPq (Brazil) and bibliographic databases: Lilacs (Bireme/OPS) and PubMed. We observed that the number of papers published increased after the participation in one of these courses. Although other factors may influence the production, such initiatives stimulate scientific communication.

Keywords: scientific communication and diffusion; information literacy; training courses; papers writing; public health.

\title{
Resumen
}

Este artículo es resultado de estudio que tiene el objetivo de identificar si la mejora en el conocimiento sobre como escribir artículos científicos, reforzada por los cursos de escritura científica, ha facilitado para el investigador (autor) la publicación de sus artículos. Entendemos que la relevancia del estudio se debe al reconocimiento de la importancia de inversiones en cursos de capacitación para investigadores que permitan ampliar la divulgación científica. Identificamos la producción bibliográfica de participantes de cursos de redacción científica y comparamos el número de publicaciones del participante antes y después del curso. Se han utilizado dos fuentes de información a la disposición del público para identificar la producción bibliográfica, científica y técnica, del investigador: la Plataforma Lattes do CNPq (Brasil); y base de datos bibliográficos: Lilacs (Bireme/OPAS) y PubMed. Se observó que el número de artículos publicados fue mayor después de la participación en el curso. Aunque otros factores puedan influir en la producción, tales iniciativas estimulan la divulgación científica.

Palabras clave: comunicación y divulgación científica; alfabetización informacional; cursos de capacitación; escritura de artículos; salud pública.

\begin{abstract}
Contribuição dos autores:
Concepção e desenho do estudo: Profa. Dra. Angela Maria Belloni Cuenca, Dulcenea de Paula, Prof. Dr. Ivan França Júnior; Aquisição dos dados: Profa. Dra. Angela Maria Belloni Cuenca, Dulcenea de Paula;

Análise dos dados: Profa. Dra. Angela Maria Belloni Cuenca, Dulcenea de Paula, Prof. Dr. Ivan França Júnior; Redação do manuscrito: Profa. Dra. Angela Maria Belloni Cuenca;

Revisão crítica do conteúdo intelectual: Profa. Dra. Angela Maria Belloni Cuenca, Prof. Dr. Ivan França Júnior.
\end{abstract}

Declaração de conflito de interesses: Não há

Fontes de financiamento: Projeto financiado pela Reitoria da USP processo nº.2014-486

Considerações éticas: Não há

Agradecimento/Contribuições adicionais: nenhum

Histórico do artigo: Submetido: 19.jul.2016 | Aceito: 05.jun.2017 | Publicado: 29.set.2017

Apresentação anterior: não houve

Licença CC BY-NC atribuição não comercial. Com essa licença é permitido acessar, baixar (download), copiar, imprimir, compartilhar, reutilizar e distribuir os artigos, desde que para uso não comercial e com a citação da fonte, conferindo os devidos créditos de autoria e menção à Reciis. Nesses casos, nenhuma permissão é necessária por parte dos autores ou dos editores. 


\section{Introdução}

Cientistas de todo o mundo, pressionados a publicar seus achados em revistas científicas de qualidade vêm recorrendo aos serviços de edição de manuscritos e suas instituições promovendo intervenções para esse fim $^{1-3}$. No entanto, nenhum serviço de edição ou níveis de treinamento podem melhorar conteúdos medíocres, linguagens inadequadas, dados mal descritos, entre outras limitações de autores inexperientes 4 . Para tormento do autor, junta-se o fato de que, décadas atrás, a ordem era publicar e, agora, impera o "ser citado"5. Ter seu achado embasando novas pesquisas equivale a tê-lo publicado em uma revista de impacto na ciência. Agrega-se a esse cenário, a explosão mundial da produção científica, uma indústria, em crescimento, de artigos, livros, teses, eventos entre outras publicações numa quantidade que quase foge do controle do cientísta6. Isto exige que os pesquisadores desenvolvam cada vez mais competências e habilidades para suas atividades, inclusive as voltadas à publicação.

Nesse cenário proliferam-se diversos provedores de serviços, de publishers para facilitar e agilizar a disseminação científica. Um dos fortes investimentos tem sido a capacitação de autores para aprimoramento da escrita científica. Em revisão sobre o assunto, McGrail e colaboradoras7 identificaram modalidades de capacitação, desde cursos curtos, intervenções a longo prazo até grupos de apoio, ou coaching, sobre como publicar artigos científicos. Aí se enquadram além de cursos, seminários, workshops e oficinas promovidos por especialistas ${ }^{8}$ - universidades, sociedades científicas - destinados a capacitar o pesquisador para a redação de artigos.

Iniciativas de capacitação para a escrita científica, como cursos curtos, podem ser efetivos e trazerem resultados positivos na produção bibliográfica.

No estudo aqui apresentado buscou-se avaliar se cursos curtos de escrita científica, com conteúdo voltado para a adequação de linguagem, estrutura do artigo, boa descrição do método e dos resultados, além de uma discussão bem feita, podem facilitar a publicação de artigos dos pesquisadores (autores) que deles participaram.

\section{Método}

Foi feito um estudo descritivo, comparando-se a produção científica de participantes de cursos de escrita científica, na modalidade curso curto (de difusão universitária) antes e após a sua participação. O curso avaliado foi um dos integrantes do Programa de Verão da Faculdade de Saúde Pública (FSP) da Universidade de São Paulo (USP), ministrado no período de 2010 a 2013.

\section{Caracterização do curso}

No Brasil, uma das iniciativas para melhorar a habilidade na escrita científica, tem sido promovida pela Universidade de São Paulo (USP), via ações de Cultura e Extensão, oferecendo cursos intensivos de curta duração, para ajudar docentes, pesquisadores, pós-graduandos e graduandos a colocarem seus resultados na forma de um artigo científico e assim o submeterem a revistas arbitradas, de impacto na literatura nacional e internacional. Em uma de suas unidades da área da Saúde, a USP oferece cursos curtos, abertos aos interessados de todo o Brasil, no chamado Programa de Verão da Faculdade de Saúde Pública. Um deles é para o aprimoramento na escrita de artigos científicos - Bases teóricas e metodológicas para a escrita científica.

Esse curso, na modalidade difusão (24h), é oferecido anualmente, no período das férias escolares (janeiro e fevereiro). O único requisito exigido para dele participar é estar cursando ou ter finalizado graduação em área de interesse para a saúde pública. Segue o modelo lógico de Volpatog, cujo conteúdo aborda desde as bases teóricas para fundamentação da pesquisa, passa pelos meandros da publicação científica e segue até a prática da revisão por pares (Quadro 1). 
Quadro 1 - Estrutura do curso Bases Teóricas e Metodológicas para a Escrita Científica, oferecido no Programa de Verão da FSP/USP de 2010 a 2013, com duração de 24 cada.

\begin{tabular}{|l|l|}
\hline Tópicos & Abordagem \\
\hline $\begin{array}{l}\text { Bases teóricas para a } \\
\text { publicação científica }\end{array}$ & $\begin{array}{l}\text { A fundamentação empírica; objetividade científica; interpretação e leis gerais; } \\
\text { O que publicar? Por que publicar? Qual a novidade do estudo? }\end{array}$ \\
\hline Publicação científica & $\begin{array}{l}\text { Evolução do conceito de comunicação científica; publicações impressas e online; o } \\
\text { processo de publicação. }\end{array}$ \\
\hline Por que não publicamos? & A falta de tempo e de conhecimento; o medo de errar; causas de rejeição. \\
\hline Onde publicar? & $\begin{array}{l}\text { Idioma; conceitos sobre qualidade científica; número de publicações; qualidade } \\
\text { do periódico; aceitação pela comunidade científica; autoavaliação do impacto das } \\
\text { publicações. }\end{array}$ \\
\hline Entendimento e redação & $\begin{array}{l}\text { Estruturação lógica; a lógica do artigo científico; essência dos tipos de pesquisa; } \\
\text { dinâmica da redação: o estilo científico; redija pensando no leitor. }\end{array}$ \\
\hline Bases metodológicas para a \\
redação científica & $\begin{array}{l}\text { Princípio da parcimônia; a lógica do teste de hipóteses; variáveis experimentais; } \\
\text { fundamentando o texto científico: amostragem; número e unidade de réplicas; } \\
\text { grupos de controle; reduzindo a subjetividade; dependência e independência entre } \\
\text { variáveis; técnicas sofisticadas; quantificação dos dados; rudimentos de estatística } \\
\text { necessários para a redação científica. }\end{array}$ \\
\hline Autoria & Questão do plágio e integridade na ciência. \\
\hline A estrutura do texto científico & $\begin{array}{l}\text { As partes do texto científico: o resumo; as conclusões; os resultados; o material e } \\
\text { métodos; a discussão; a introdução; as referências; o título; as demais partes do } \\
\text { texto. }\end{array}$ \\
\hline A prática da redação & O estudante como peer review. \\
\hline
\end{tabular}

Fonte: Adaptado de Volpato, 2011

\section{População do estudo}

Foram elegíveis para o estudo 211 participantes dos cursos de escrita científica do Programa de Verão da FSP/USP durante o período de 2010 a 2013.

Cabe informar que, inicialmente, o número de participantes totalizava 217; porém, seis foram excluídos devido à impossibilidade de identificação de suas produções, pelos seguintes motivos: dois não possuíam cadastro na Plataforma Lattes do CNPq; dois tinham seus currículos na Plataforma Lattes desatualizados, com data anterior à participação no curso; e dois já haviam participado do curso mais de uma vez.

\section{Coleta de dados}

Foram utilizados dois tipos de fontes de informação de acesso público para identificar e conhecer a produção bibliográfica, científica e técnica, do participante: a) currículo na Plataforma Lattes do CNPq; e b) fontes de dados bibliográficos nas bases Lilacs e PubMed, ambas da área da saúde.

Em relação aos procedimentos, no currículo Lattes foram identificados os artigos publicados pelo egresso, após pré-teste com 10 egressos para detectar possíveis dificuldades na coleta das informações e, principalmente, para determinar as categorias de análise.

As bases de dados bibliográficas da área da saúde - Lilacs e PubMed - foram consultadas para confirmação da produção registrada no Lattes e para coleta de publicações adicionais, não constantes no Lattes. Isso porque foi identificado no pré-teste que vários egressos não tinham nos seus Lattes informação sobre publicações, embora a data da última atualização estivesse correta.

O período estipulado para abranger os artigos a serem buscados nessas bases de dados foi de até três anos antes e depois do curso - de modo a verificar se o participante possuía a prática de publicação antes de participar do curso e se teve artigos publicados após. Portanto, o período abarcado pela busca foi desde 2008 até 2015, garantindo tempo hábil para o egresso do último curso ter publicado. 


\section{Análise dos dados}

As informações foram inseridas em planilha eletrônica com os seguintes dados: nome do participante, área acadêmica de formação, ano de participação no curso, endereço do currículo Lattes, número de artigos na base Lilacs, número de artigos no portal PubMed, número de artigos publicados antes do curso, número de artigos após a participação no curso.

\section{Resultados}

O número de artigos publicados foi maior após a participação no curso. Nesse período, os 211 egressos publicaram 716 artigos, enquanto anteriormente haviam publicado 498. Quanto à distribuição de publicações ao longo do período estudado, observa-se também aumento após o curso (Gráfico 1).

Em 2010, os 64 alunos haviam publicado antes do curso uma média de 2,79 artigos cada um; após o curso essa média foi para 3,82. Em 2011, antes do curso os 81 alunos publicaram, em média, 2,91 artigos cada um; depois do curso, 4,23. Em 2012, 34 alunos publicaram antes do curso um artigo, em média, cada um; após o curso, 2,76. Em 2013, os 32 alunos haviam publicado 49 artigos antes do curso, em média 1,53 cada; e após, caiu para 1,06.

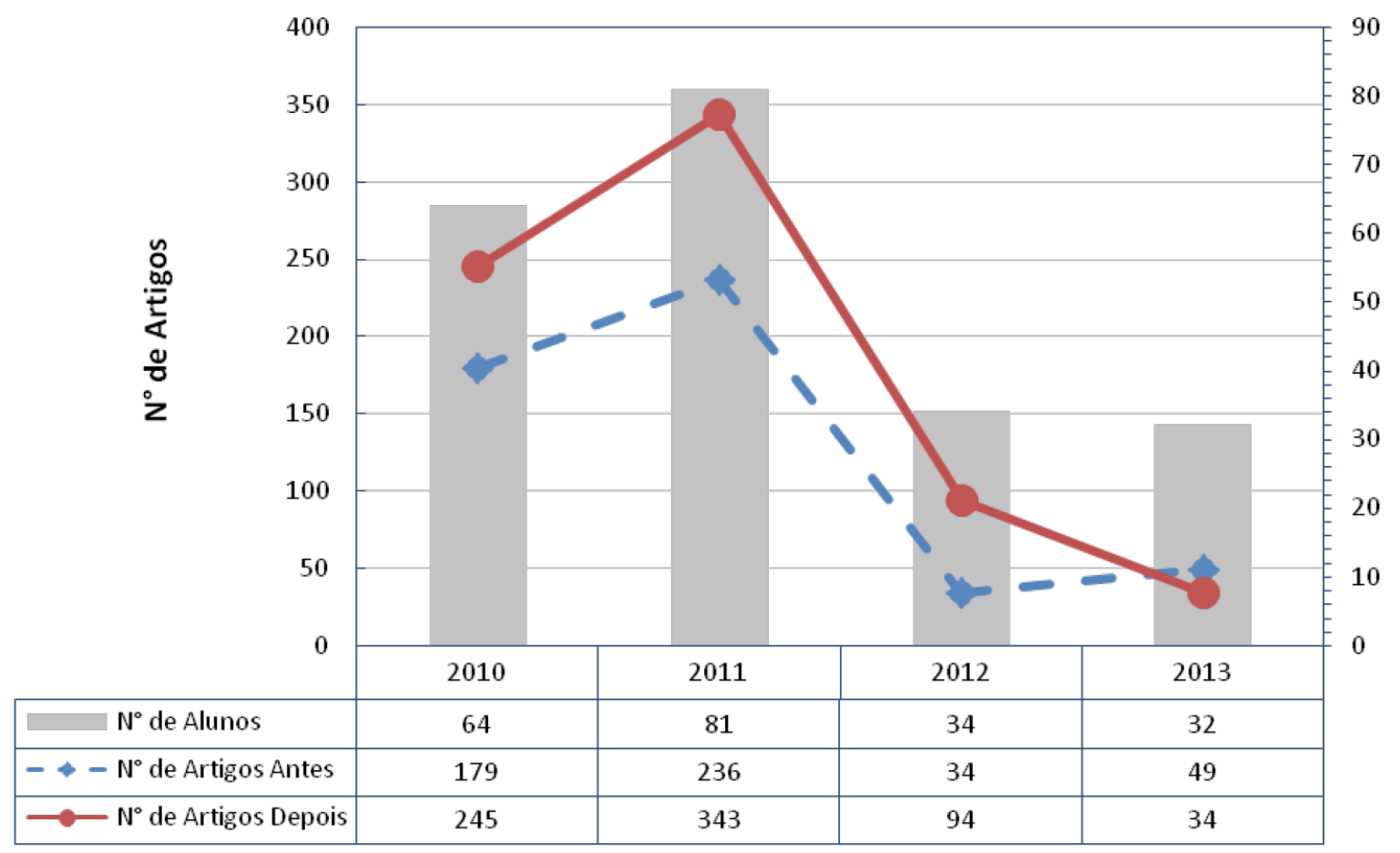

Gráfico 1 - Artigos pré e pós curso de escrita, por ano de publicação dos egressos do Programa de Verão da FSP/USP 2010 a 2013 Fonte: Os autores (2017).

Cabe esclarecer que, no ano de 2011, houve um número maior de participantes porque a FSP ofereceu dois cursos de escrita no mesmo Programa.

Ainda com o foco no Gráfico 1, cabem observações sobre dois aspectos: o número de participantes no curso vai diminuindo ao longo do tempo; e os participantes de 2013 tiveram menos publicações após o 
curso. O primeiro aspecto pode ser atribuído ao atendimento de uma demanda reprimida, existente no início, por cursos dessa natureza; melhor dizendo, à medida em que essa demanda foi sendo atendida, o número de participantes permaneceu estável. Isto porque os pós-graduandos representam a maioria dos participantes e o número de alunos que ingressa anualmente tende a ser o mesmo.

Quanto ao segundo aspecto, o menor número de publicações após o curso pode ser atribuído ao fato de ele ter ocorrido em 2013 e a coleta deste estudo ter sido realizada logo após (cerca de 18 meses), tempo insuficiente para se produzir e conseguir publicar uma pesquisa nas revistas científicas.

\section{Discussão}

Aqui mostramos que houve benefício para a comunidade participante, pelo menos no que diz respeito à quantidade de publicações. Isto porque, obviamente, o aprimoramento na escrita não é fator exclusivo para o aumento na produção científica. Outros aspectos influenciam sobremaneira a produção de um cientista, como a pressão por publicar, disponibilidade de fomento à pesquisa, infraestrutura adequada, entre outros. A real contribuição de cursos, como o aqui analisado, ou seja, se foram efetivos e eficazes, deve levar em conta a qualidade do que foi publicado e não somente seus dados numéricos.

No entanto, investimentos no aprimoramento da habilidade para a pesquisa científica (scientific literacy) têm sido descritos como importante ferramenta da investigação científica11, desde a fase de graduação dos estudantes11, como forma de conhecerem todo o processo de publicação e revisão por pares ${ }^{12}$ Sobre este aspecto, cabe ressaltar que, além de pesquisadores e pós-graduandos, estudantes de graduação também podem participar do curso da FSP/USP como forma de estímulo, principalmente os envolvidos nos projetos de iniciação científica.

Quanto à avaliação de cursos, retomando McGrail e colaboradoras7 ${ }^{7}$, cursos curtos de escrita científica tendem a ter resultados mais imediatos. No entanto, pouco se conhece sobre a eficiência e eficácia de cursos dessa natureza.7 As avaliações são restritas ao período imediatamente após seu término, frequentemente com boas impressões e recomendações de maior investimento em iniciativas como essa, geralmente abordando aspectos como conteúdo, metodologia didática, infraestrutura física, entre outros. McGrail e colaboradoras ${ }^{7}$ mencionam que os egressos saem motivados a publicar seus achados e as universidades se beneficiam, pois com isso têm maior produtividade.

Cursos como esses desmistificam o processo da escrita ${ }^{8}$ e podem ser ministrados para qualquer área do conhecimento, em qualquer nível e contexto, constituindo em aprendizado ao longo da vida ${ }^{12}$.

\section{Conclusão}

A pressão para se publicar achados de impacto na ciência, em uma época de grande produção científica, gera demanda de que os estudiosos escrevam bem e, assim tenham seus artigos publicados. Cursos de escrita científica favorecem a produção bibliográfica de seus egressos. Com isso, embora o pesquisador no Brasil tenha inúmeras outras atividades acadêmicas, podemos inferir que o aprimoramento da habilidade na escrita científica facilita a publicação, pelo pesquisador (autor), de seus artigos. No entanto, cabe lembrar que outros importantes fatores influenciam no impacto da ciência como, por exemplo, a qualidade da produção; porém, faltam estudos que avaliem esse atributo.

Entendemos que a relevância do estudo aqui apresentado se deva à possibilidade do reconhecimento, pelas instituições brasileiras, da importância de investimento no aprimoramento do pesquisador para a divulgação científica. 


\section{Referências}

1. Aebi M.The impact fator:publish, be cite dor perish. Eur Spine J. 2002; 11 Supl 1.

2. Cope B, Kalantzis M, Abd-El-Khalich F, Bagley E. Science in writing: learning scientific argument in principle and practice. E-Learning Digit Media. 2013;10(4):420-38.

3. Cox BG, Schatz DA, Van Mierop LHS. Scientific writing courses for pediatrics fellows. Acad Med.1990; 65(10):652-3.

4. Kaplan K. A helping hand. Nature. 2010; 468(7324):721-3.

5. Glaser RE. Design and assessment of an assignment-based curriculum to teach scientific writing and scientific peer review. Gateways. 2014; 7(2):85-104.

6. Lyons BP, Elmedni B. Writing skill development for graduate students: workshop intervention usisng a student-centering learning approach. J Educ Soc Policy. 2015; 2(1):38-48.

7. McGrail M, Rickard CM, Jones R. Publish or perish: a systematic review of interventions to increase academic publication rates. High Educ Res Develop. 2006; 25(1):19-35.

8. Marques F. Escreva bem ou pereça. Pesquisa Fapesp. 2011;(182):34-9.

9. Volpato G. O método lógico para redação científica. Reciis [Internet]. 2015 [citado 2015 set. 03]; 9(1):1-14. Disponível em: https://www.reciis.icict.fiocruz.br/index.php/reciis/article/view/932/1577

10. Volpato GL. Método lógico para redação científica. Botucatu: Best Writing; 2011.

11. McNutt M. Improving scientific communication. Science. 2013; 342(6154):13.

12. Pearson PD, Moje E, Greenleaf C. Literacy and Science: each in the service of the other. Science. 2010; 328:459-63. 